\title{
Urethral dysfunction due to alloxan-induced diabetes. Urodynamic and morphological evaluation ${ }^{1}$
}

\author{
Ana Beatriz Gomes de Souza Pegorare ${ }^{\mathrm{I}}$, Marco Antônio Gonçalves ${ }^{\mathrm{II}}$, Carla Adelino Suaid ${ }^{\mathrm{III}}$, Antonio Antunes Rodrigues Junior ${ }^{\mathrm{IV}}$, \\ Silvio Tucci Jrv ${ }^{\mathrm{v}}$, Haylton Jorge Suaid ${ }^{\mathrm{VI}}$ \\ DOI: http://dx.doi.org/10.1590/S0102-86502014000700008 \\ IFellow PhD degree, Postgraduate Program in Surgery, Department of Surgery and Anatomy, School of Medicine of Ribeirao Preto, University of Sao \\ Paulo (FMRP-USP), Ribeirao Preto-SP, Brazil. Surgical procedures, acquisition and analysis of data, manuscript writing.

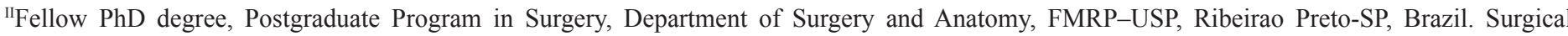 \\ procedures, analysis of data, manuscript writing. \\ IIIFellow PhD degree, Postgraduate Program in Surgery, Department of Surgery and Anatomy, FMRP-USP, Ribeirao Preto-SP, Brazil. Interpretation of data.

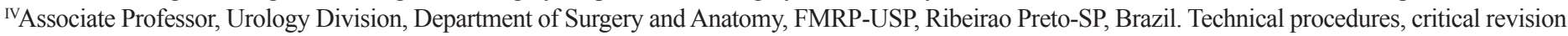 \\ ${ }^{v}$ Associate Professor, Urology Division, Department of Surgery and Anatomy, FMRP-USP, Ribeirao Preto-SP, Brazil. Critical revision. \\ ${ }^{V}$ Associate Professor, Urology Division, Department of Surgery and Anatomy, FMRP-USP, Ribeirao Preto-SP, Brazil. Design the protocol, final \\ approval of the version to be published.
}

\section{ABSTRACT}

PURPOSE: To evaluate the effect of short and long term alloxan-induced diabetes on bladder and urethral function of female rats, and also describing its correlated morphological alterations.

METHODS: Thirty five female rats were divided into three groups: $\mathrm{G} 1(\mathrm{n}=9)$, control group; $\mathrm{G} 2(\mathrm{n}=17)$, six weeks alloxan-induced diabetic rats; G3 (n=9), 20 weeks alloxan-induced diabetic rats. Functional evaluation was performed by cystometry and simultaneous measurements of the urethral pressure during bladder filling and voiding. Morphological evaluation was also performed with measurement of bladder and urethral fibrosis and collagen content and thickness of lamina propria and smooth muscle layers.

RESULTS: The peak bladder pressures and contraction amplitudes were decreased in $100 \%$ and $47 \%$ of the G3 and G2 groups respectively, when compared to control. Bladder overactivity was observed in $53 \%$ of the G2 group.

CONCLUSION: Alloxan-induced diabetes urethropathty in female rat was associated to bladder morphological alterations as higher thicknesses of it lamina propria, detrusor and adventicea.

Key words: Diabetes Mellitus. Urinary Bladder. Urethra. Urodynamics. Rats, Wistar. 


\section{Introduction}

Lower urinary tract dysfunction is among the most common and costly consequences of diabetes mellitus (DM), and the bladder has been the primary focus of study for these complications ${ }^{1}$.

The classic description of diabetic cystopathy comprises decreased bladder sensation, impaired detrusor contractility and impaired bladder emptying with resultant elevated post-void residual urine volume. In addition to these functional impairments, DM also induced alterations in bladder mass, tissue composition, and compliance ${ }^{2,3}$. Recent findings support the hypothesis that muscle atrophy and fibrosis occur in aging and these processes are more severe in the diabetic rat urethras. Also associated a suggest that bladder dysfunction of the diabetic patients correlates with the underlying morphological alterations of detrusor muscle layer and ratio of collagen I and III more than with diabetic autonomic neuropathy ${ }^{4,5}$.

In this study, we aimed to evaluate the effects of DM at six and 20 weeks after DM induction on urodynamics evaluation and also, to verify the morphologic alterations correlation with the contribution of DM to the voiding dysfunction seen in diabetic rats.

\section{Methods}

All procedures involving animals were in accordance with the guidelines established by COBEA (Brazilian College on Animal Experimentation) and approved by the Ethics Committee on Animal Experimentation of School of Medicine of Ribeirao Preto (CETEA) number 035/2009.

Thirty five female Wistar rats (Rattus norvegicus albinus) of the same age weighing on average $250 \mathrm{~g}$ were used. The animals were house in collective cages at room temperature on sunlight cycle, with water and ration ad libitum. Diabetes mellitus (DM) was induced at random in 26 rats by a single intraperitoneal injection of alloxan, $40 \mathrm{mg} / \mathrm{kg}$ body weight, dissolved in $0.05 \mathrm{M}$ sodium citrate, $\mathrm{pH} 4.5$, at $4^{\circ} \mathrm{C}$. Blood glucose level was determined by the glucose oxidation method (Accu-Check Complete System, Roche) 72 hours after alloxan administration. All diabetic animals had a glycemia level of more than $300 \mathrm{mg} / \mathrm{dl}$.

The animals were divided in three groups - G1: six week control; G2: six week alloxan induced diabetes; G3: 20 week alloxan induced diabetes. Diabetes was induced by a single intraperitoneal injection of alloxan $(40 \mathrm{mg} / \mathrm{kg})$ dissolved in 0.05 $\mathrm{M}$ sodium citrate, $\mathrm{pH} 4.5$, at $4^{\circ} \mathrm{C}$. Controls were identically treated, but buffer was injected instead of the alloxan solution.
As inclusion criteria for diabetic animals and the hyperglycaemic state was confirmed through blood glucose testing 72 hours later, by the glucose oxidation method (Accu-Check Complete System, Roche). Functional and morphological evaluation were performed at six and twenty weeks and diabetic animals were compared to control ones.

\section{Urodynamic evaluation}

The evaluation was performed after intraperitoneal anesthesia with urethane $(1.25 \mathrm{mg} / \mathrm{kg} \text { weight })^{6}$. After suprapubic median laparotomy, a PF-50 polyethylene catheter was introduced and fixed on the bladder fundus and exteriorized by counter- opening in the dorsolateral region. The absence of local extravasation and catheter permeability were checked ${ }^{7}$ and cystometry was performed through this catheter.

The parameters analyzed in the cystometry evaluation were: maximum micturition pressure (MMP), bladder contraction frequency $(\mathrm{F})$, bladder contraction intensity $(\mathrm{CI})$, time of bladder filling (TF), and time of bladder contraction (TC). Bladder capacity (C) was obtained by multiplying TF by flow.

For the determination of urethral pressure (UP) a polyethylene catheter $(0.64 \mathrm{~mm}$ outer diameter and $0.50 \mathrm{~mm}$ inner diameter) was positioned at the level of the bladder neck and connected in "Y" to the system for pressure recording. Saline solution was infused through the catheter with an infusion pump at a flow of $0.75 \mathrm{ml} / \mathrm{minute}$.

The cystometric and urethral pressure recordings were obtained with a Dynamed ${ }^{\circledR}$ instrument, model Dynapack MPX816 , with a Y connection to the cystostomy and urethral catheters and to the continuous infusion pump regulated at a flow of 0.75 $\mathrm{mL} / \mathrm{min}$, which permitted a simultaneous continuous recording of vesical and urethral pressure. The transducer for the recording of urethral pressure was positioned at the level of the pubic symphysis. Data were collected for 10 minutes and analyzed with the UroMaster II software.

Each animal was submitted to urodynamic evaluation for 30 minutes and sacrificed while still under the effect of anesthesia.

Data were analyzed based on the following parameters: mean urethral pressure during detrusor contraction (PUC) and mean urethral pressure during bladder filling (UPF).

\section{Statistical analysis}

The obtained values of parameters of cystometry and urethral pressure recordings were evaluated using nonparametric 
Kruskal Wallis with Dunn post-test, considering significant differences with a $\mathrm{p}<0.05$. The results were expressed as mean \pm standard deviation (SD). Calculations were made using GraphPad Prism 3.02.

Before death, the rats were sedated with urethane $(1.25$ $\mathrm{mg} / \mathrm{kg}$ ), weighed, and blood samples were taken for glucose quantification. Infections were excluded by culturing urine samples collected by bladder puncture. The rats were killed by intraperitoneal Injection of pentobarbital (200 mg/kg). The proximal portions of the urethra and anterior portions of the bladder fundus were removed and fixed at the optimal length in neutral buffer formalin (10\%) for 24 hours. The paraffin-embedded tissues were sectioned at a 5-ưm thickness and stained for muscle layer thickness, connective tissue, and collagen analysis. The experimental protocol followed the guidelines of the experimental ethics committee of our institution.

\section{Histologic sample manipulation}

The stained sections were analyzed with a Carl Zeiss-2 Axiskop plus microscope (Carl Zeiss, Jena, Germany) with 40 magnification. Images were captured at a 1300 - 1030 pixel definition through an analog-digital camera (JVC TK-1270u, JVCUSA, Wayne, NJ) connected to the microscope and a computer. The total thickness and muscular layer thickness were measured on images stained with hematoxylin-eosin. Three linear measures from different quadrants of each image were performed in each field, with the results represented in microns. The connective tissue area, total area, and muscle area were measured on images stained with Masson's trichrome stain.

\section{Morphological analysis}

The total thickness and muscular layer thickness were measured on images stained with hematoxylin-eosin. Three linear measures from different quadrants of each image were performed in each field, with the results represented in microns. The connective tissue area, total area, and muscle area were measured on images stained with Masson's trichrome stain.

\section{Results}

After six weeks of induction of diabetes, the 17 animals monitored, eight animals were in the phase of decompensation of the detrusor muscle, evolving with bladder hipoactivity. These animals were separated and designated groups G2b. For the end of the study could take the data from this intermediate group for comparisons.

The diabetic rats (groups 2 and 3) demonstrated a lower weight and greater glucose levels at death than the control rats (group 1). All urinary microbiologic cultures were negative before death.

\section{Functional evaluation}

No significant difference was observed between groups in the analysis of MMP $(p=0.633), B F(p=0.746), F(p=0.5717)$ or $\mathrm{TF}(\mathrm{p}=0.07)$ (Table 1$)$.

TABLE 1 - Functional variables analyzed in the groups.

\begin{tabular}{cccccc}
\hline VARIABLES & G1 & G2 & G2B & G3 & p \\
\hline MMP & 23.5 & 24.5 & --- & --- & $\mathbf{0 . 6 3 0 3}$ \\
\hline BC & 0.48 & 0.37 & --- & --- & $\mathbf{0 . 1 0 0 3}$ \\
\hline F & 1.5 & 1.8 & --- & --- & $\mathbf{0 . 5 7 1 7}$ \\
\hline TC & 11.5 & 18.1 & --- & --- & $\mathbf{0 . 0 2 7 8 *}$ \\
\hline TF & 37.5 & 29.6 & --- & --- & $\mathbf{0 . 7 4 6 0}$ \\
\hline PUC & 10.5 & 16.0 & 21.1 & 27.0 & $\mathbf{0 . 0 0 1 2 *}$ \\
\hline UPF & 8.3 & 13.5 & 25.6 & 25.5 & $\mathbf{0 . 0 0 0 1 *}$ \\
\hline
\end{tabular}

*Kruskal-Wallis non-parametric test

However, in 20 weeks alloxan-induced diabetes rats, there was a significant reduced bladder pressure accomplish poor emptying, and detrusor smooth muscle failure. That's why we couldn't compare the following parameters with the others groups.

\section{Bladder contraction time (TC)}

There was a significant increase in this variable in the group of diabetic rats. The post hoc Dunn test revealed that this difference occurred between G1 and G2 animals ( $p=0.0278$ )*.

Urethral pressure during detrusor contraction (PUC). There was a significant difference between $\mathrm{G} 1$ and $\mathrm{G} 2 \mathrm{~B}(\mathrm{p}=0.001)$, group G1 and G3 (p=0.001) (Figure 1). 


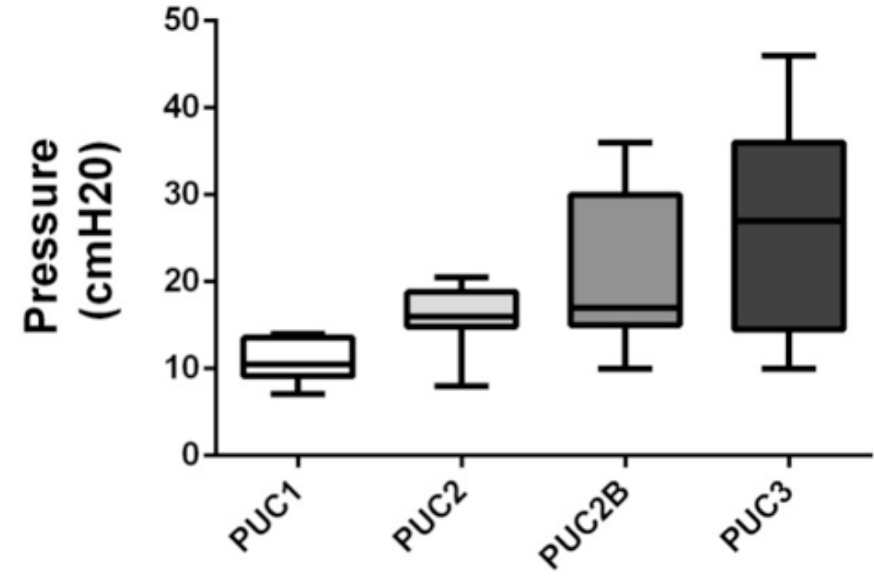

FIGURE 1 - Comparison of urethral pressure during detrusor contraction in $\mathrm{G} 1, \mathrm{G} 2, \mathrm{G} 2 \mathrm{~B}$ e G3 in $\mathrm{cmH}_{2} 0$ (Post hoc Dunn G1xG2B ( $<<0.001$ ); G3xG1 $(\mathrm{p}<0.05)$.

\section{Urethral pressure during bladder filling (UPF)}

There was a significant difference between G1 and G2B $(\mathrm{p}=0.0001)^{*}$ and between G1 and G3 ( $\left.\mathrm{p}=0.001\right)$ (Figure 2).

It is possible to see the variations in urethral pressure during the micturition cycle in normal rats (Figure 3a) and 6-weeks diabetic rats with elevated urethral pressure during

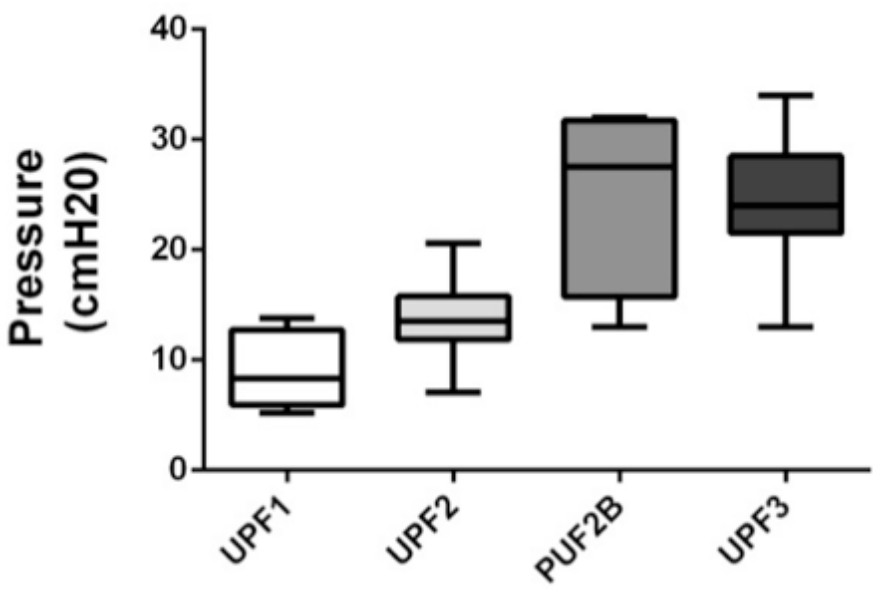

FIGURE 2 - Comparison of urethral pressure during detrusor filling in $\mathrm{G} 1, \mathrm{G} 2, \mathrm{G} 2 \mathrm{~B}$ e $\mathrm{G} 3$ in $\mathrm{cmH}_{2} 0$ (Post hoc Dunn G1xG2B ( $<<0.001$ ); G3xG1 $(\mathrm{p}<0.05)$.

voiding (Figure 3b). However, urodynamic evaluation of a sixweeks diabetic rat (G2B) demonstrating dysfunctional voiding cycle. It is not possible to observe the relaxation of the urethra during voiding of the bladder (Figure 4a). Urodynamic study of a 20 weeks female diabetic rat can be observed in the tracings of the urodynamic recordings high urethral pressure, impaired detrusor contractility and impaired bladder emptying (Figure 4b).
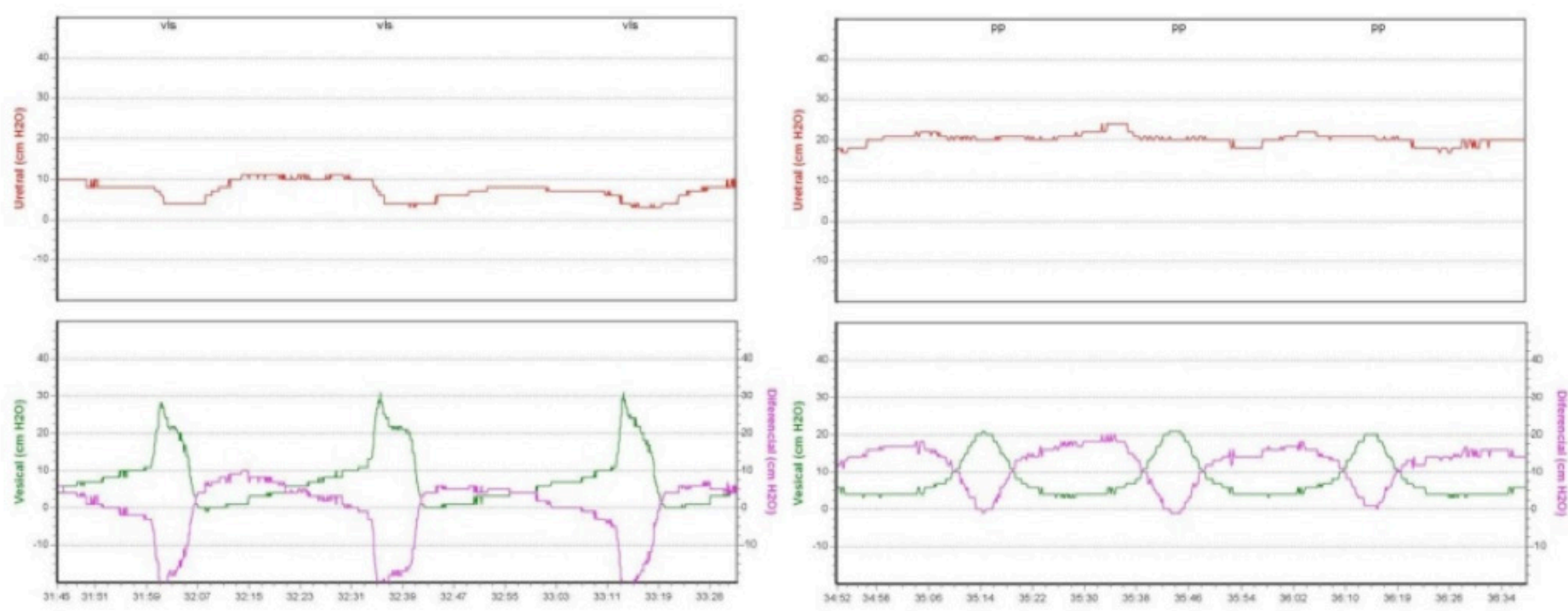

FIGURE 3 - a) Urodynamic evaluation of a healthy rat (G1) demonstrating voiding cycle normal. Can observe the relaxation of the urethra during the emptying phase. b) Urodynamic study of a diabetic rat (G2) showing elevated urethral pressure during voiding cycle. 

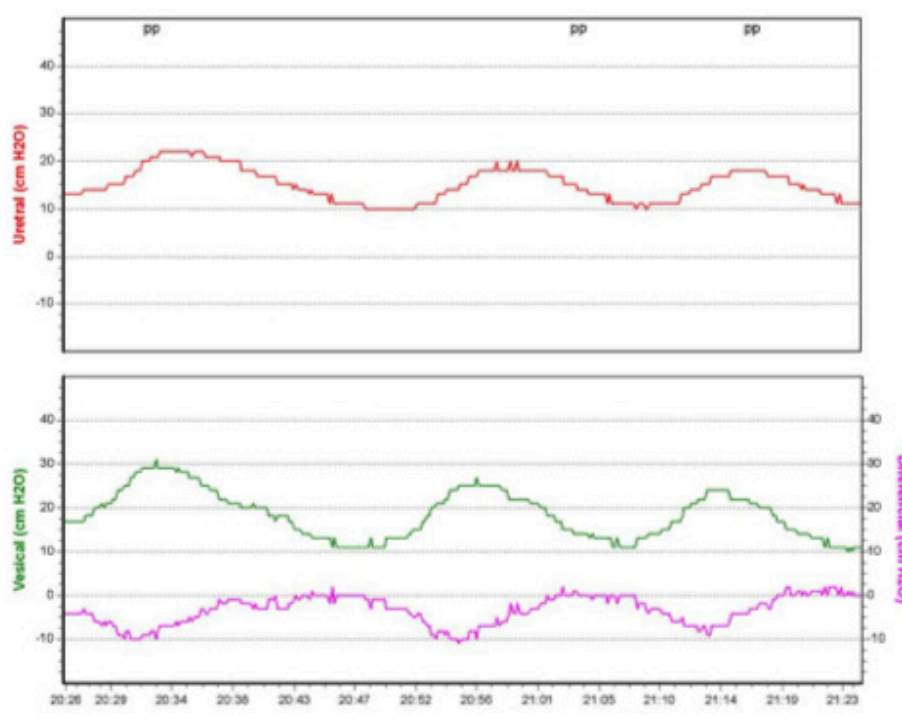
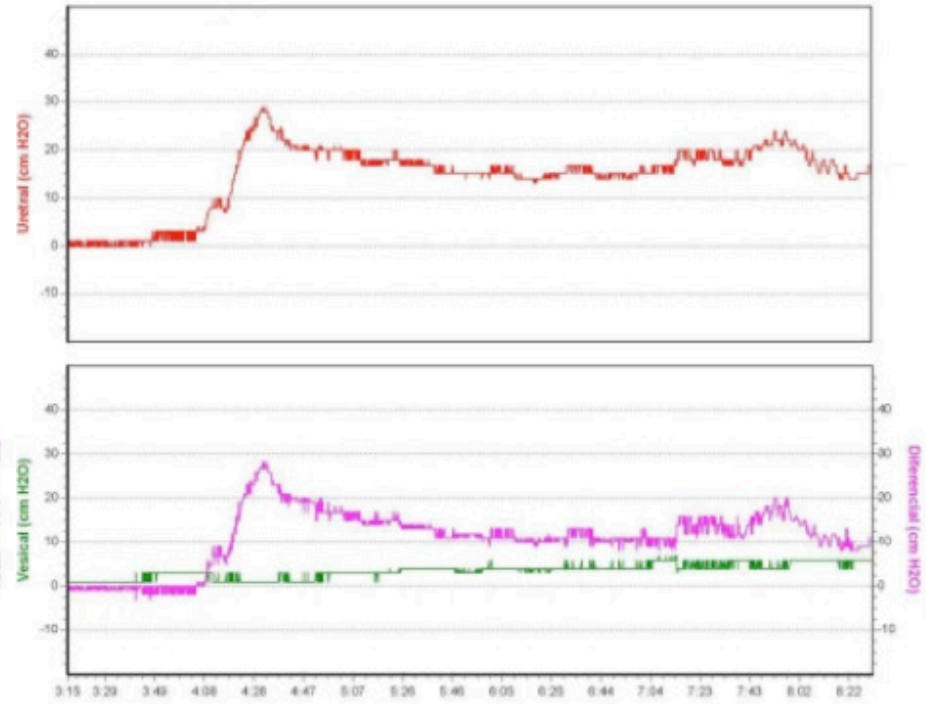

FIGURE 4 - a) Urodynamic evaluation of a diabetic rat (G2B) demonstrating dysfunctional voiding cycle. It is not possible to observe the relaxation of the urethra during voiding of the bladder. b) Urodynamic study of a 20 weeks female diabetic rat. It is possible to see high urethral pressure, impaired detrusor contractility and impaired bladder emptying.

\section{Bladder and urethral morphological analyses}

The bladders from groups 3 and 2 had greater values of total thickness (TTB) than did the control group $(\mathrm{p}<0.0001)$ ranging between $552.96 \mu \mathrm{m}$ (G1) and $898.93 \mu \mathrm{m}$ (G3). The bladder muscle layer thickness (BMT) from groups 3 and 2 had greater values than did the control group $(\mathrm{p}<0.0001)$ ranging between $377.29 \mu \mathrm{m}$ (G1) and $603.49 \mu \mathrm{m}$ (G3) (Table 2).

TABLE 2 - Bladder and urethral morphological variables analyzed in the groups.

\begin{tabular}{ccccc}
\hline VARIABLES & G1 & G2 & G3 & p \\
\hline TTB & 552.96 & 655.49 & 898.93 & $\mathbf{0 . 0 0 0 1 *}$ \\
BMT & 377.29 & 424.36 & 603.49 & $\mathbf{0 . 0 0 0 1 *}$ \\
BCT & 161.76 & 218.05 & 281.31 & $\mathbf{0 . 0 0 1 *}$ \\
TTU & 192.75 & 224.94 & 419.58 & $\mathbf{0 . 0 0 0 1 *}$ \\
TUU & 13.73 & 12.46 & 12.17 & $\mathbf{0 . 0 1 *}$ \\
TUB & 12.12 & 13.08 & 12.64 & $\mathbf{0 . 0 0 1 *}$ \\
\hline
\end{tabular}

*Teste One way ANOVA

The total bladder connective tissue area (BCT) was larger in group 3 than in group $1(\mathrm{p}=0.001)$ ranging between 161.7629 $\mu \mathrm{m}(\mathrm{G} 1)$ and $281.31 \mu \mathrm{m}(\mathrm{G} 3)$.
The urethras from groups 3 and 2 had greater values of total thickness (TTU) than did the control group $(\mathrm{p}<0.0001)$ ranging between $192.75 \mu \mathrm{m}$ (G1) and $419.58 \mu \mathrm{m}$ (G3) (Table 2).

The urethra from groups 3 and 2 had lower thickness of the urothelium of the urethra (TUU). There was a significant difference between $\mathrm{G} 1$ and $\mathrm{G} 2(\mathrm{p}=0.01)$, group $\mathrm{G} 1$ and G3 $(\mathrm{p}=0.01)$, ranging between $13.73 \mu \mathrm{m}$ (G1) and $12.17 \mu \mathrm{m}(\mathrm{G} 3)$.

The bladder from group 3 and 2 had lower thickness of the urothelium of the bladder (TUB). There was a significant difference between $\mathrm{G} 1$ and $\mathrm{G} 2(\mathrm{p}=0.001)$, groups $\mathrm{G} 1$ and $\mathrm{G} 3$ $(\mathrm{p}=0.001)$, ranging between $12.12 \mu \mathrm{m}(\mathrm{G} 3)$ e $14.95 \mu \mathrm{m}(\mathrm{G} 1)$ (Table 2).

\section{Histological analysis}

Some histological changes were observed in the urethra, but mainly in the urinary bladder of diabetic animals with six weeks (G2) and twenty weeks (G3) compared to the control group (G1). Thus, an increase of collagen fibers and thus increasing the thickness of the lamina in the urethra was observed (Figure 5) and the bladder (Figure 6) this latter sometimes accompanied by the presence of mast cells and macrophages (Figure 7) in the perivascular area, mainly in G3. 


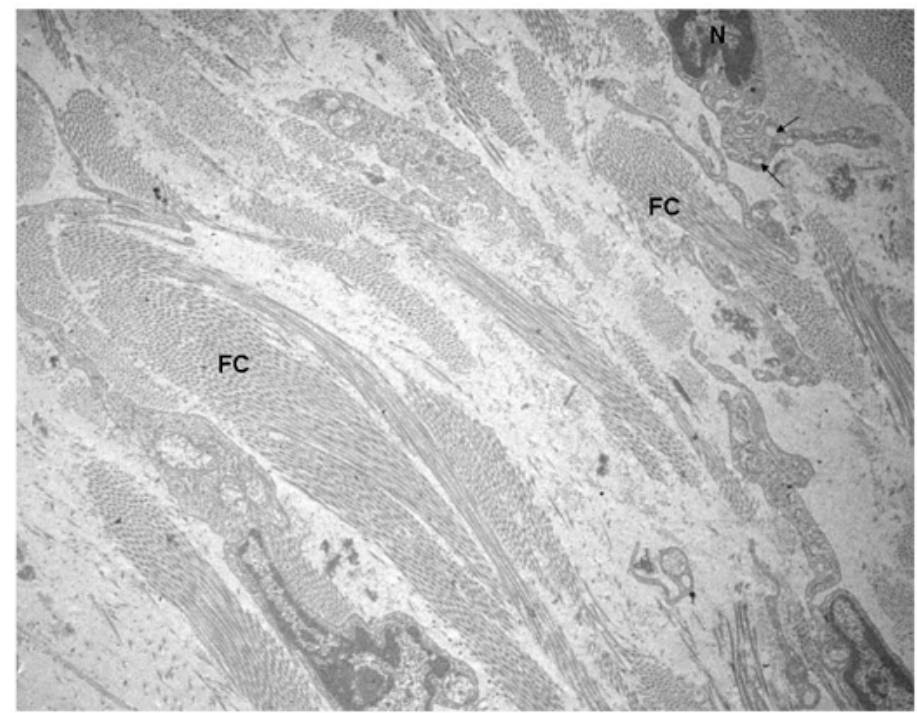

FIGURE 5 - Electron micrograph showing in detail the rise of the lamina propria in the urethra of an animal group G3. A fibroblast nucleus $(\mathrm{N})$ containing vacuoles inside $(\rightarrow)$. Collagen fibers $(\mathrm{CF})$. x6700.

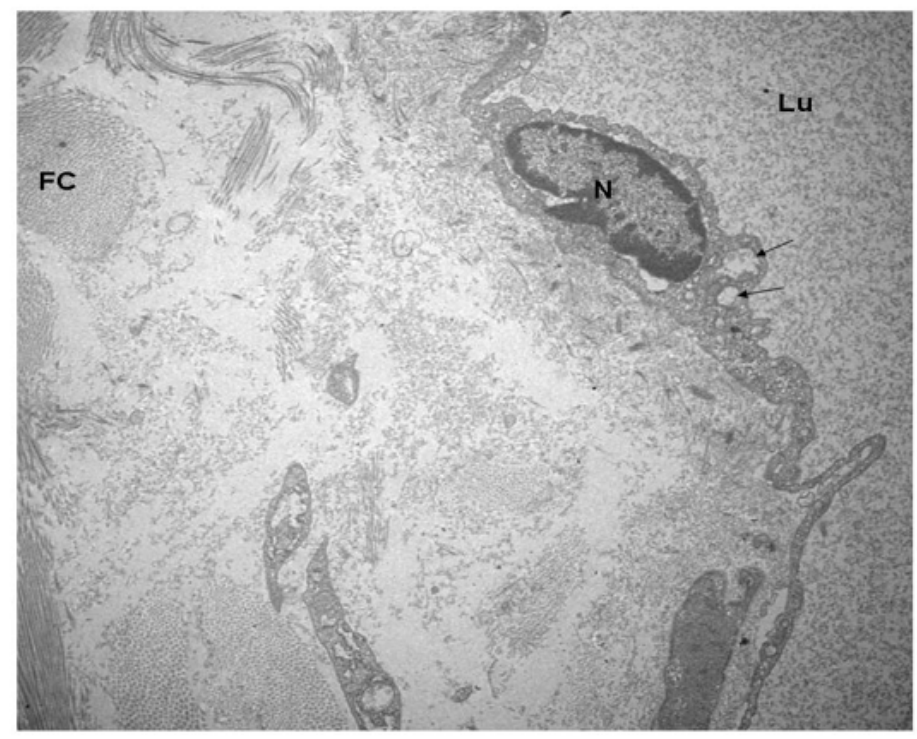

FIGURE 6 - Electron micrograph showing in detail the rise of the lamina propria in the urethra of an animal group G3. Collagen fiber (FC), an endothelial cell nucleus $(\mathrm{N})$ delimiting the lumen of a capillary $(\mathrm{Lu})$. x8000.

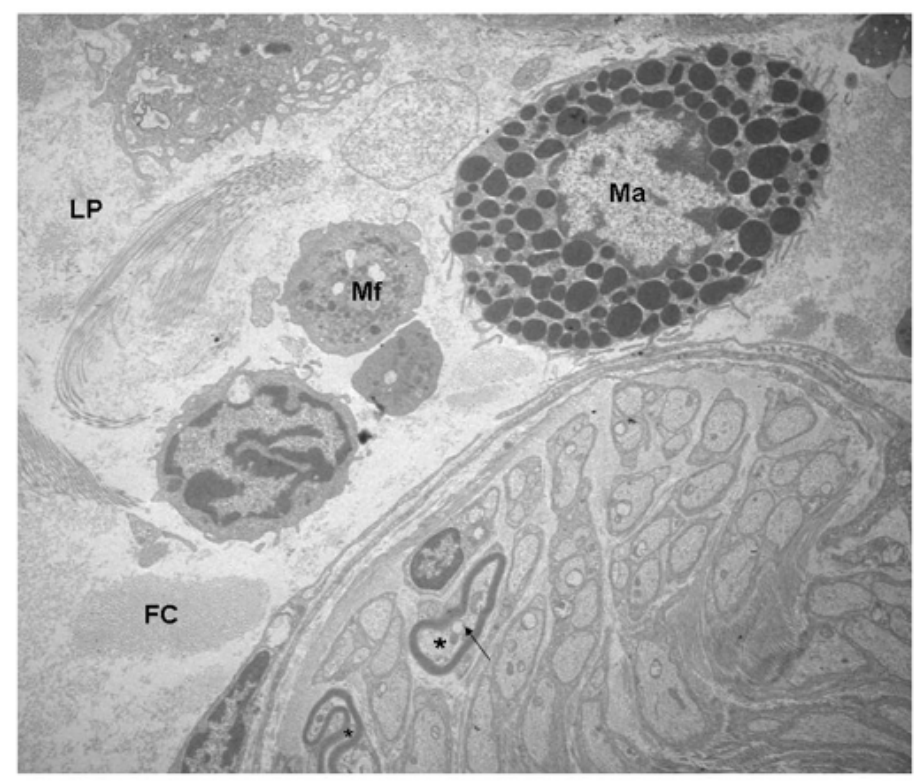

FIGURE 7 - Electron lamina propria (LP) of the bladder of an animal group G3 showing in detail the presence of a mast (Ma), and a macrophage (M) between collagen fibers (CF). Below right part of a cross section showing a nerve myelinated axons $(*)$ and unmyelinated, some to the presence of vacuoles inside $(\rightarrow)$. x 5000 .

\section{Discussion}

Diabetes mellitus (DM) has reached epidemic proportions worldwide. Complications of DM are the most important diseasespecific determinant of quality of life. Because the findings might be useful for future clinical applications, it was reasonable to investigate the effects of diabetes on the lower urinary tract of experimental models. The present study used an induced diabetic rat model. We also chose not to offer any treatment, including insulin replacement, to enhance the effects of the hyperglycemic state on the tissues we evaluated. In the present study, the efficacy of the alloxan-induced diabetic model was confirmed by the high levels of serum glucose and lower weights among the diabetic rats than among aging rats at their death ${ }^{6-13}$.

One important finding in the present study is that higher levels of urethral pressure values during bladder contraction at six weeks of alloxan-induced diabetes. Moreover, the urethral pressure proved to be significantly higher in animals with detrusor dysfunction $(\mathrm{G} 2 \mathrm{~B})$ than those who were preserved with detrusor function (G2) at six weeks of induction of diabetes.

Few studies have investigated long-term alterations in the urethra secondary to diabetes. After 20 weeks of observation, Liu et $a l .{ }^{14}$ described functional alterations of the external urethral sphincter in diabetic rats associated with atrophy of the striated muscle from the middle urethra. In contrast, short-term 
studies have de impairment. Altered urethral sympathetic and nitrinergenic responses were observed in the external urethral sphincter of streptozotocin-induced diabetic animals ${ }^{15,16}$. It is not yet known which long-term effects of these neurologic alterations cause changes in the lower urinary tract. As observed by Yang et $a l .{ }^{17}$ they probably lead to outlet obstruction. It is clear that the urethra plays an important role in long-term lower urinary dysfunction and that it should be better evaluated by additional studies $^{14-17}$. Short- and long-term diabetes-induced bladder dysfunction has been well described in published studies and has been correlated with morphologic changes. Acute detrusor muscle hypertrophy, changes in the urothelium, lamina propria, and collagen, and changes in bladder neurons and neurotransmitters have all been described ${ }^{12,19}$. Our group has previously correlated bladder dysfunction with the morphometric and collagen changes in alloxan induced diabetic rats ${ }^{18}$.

The high levels of urethral pressure during voiding in diabetic rats may be an important role in understanding the pathophysiology of diabetic cistopatia, since this finding may be correlated with a significant increase in thickness of the urethra found in diabetic rats, six weeks and especially at 20 weeks of induction (Figure 6).

In 20 weeks alloxan-induced diabetes rats group, there was observed urethral smooth muscle relaxation during reflex bladder contractions was almost completely blocked. In this group, we verify decreased bladder contractility and elevated post-void residual urine volume. The bladder dysfunction pattern seems like bladder outlet obstruction. Bladder outlet obstruction mediates both functional and structural changes in the mouse bladder.

The urinary bladder, the urothelium, the stroma and the detrusor muscle, undergo progressive pathological changes observed by electron microscopy transmission. The increase of collagen fibers in the stroma was accompanied by invasion of macrophages, neuronal degeneration and the presence of mast cells (Figure 7). There was observed a decrease in the thickness of the urothelium of the urinary bladder in diabetic rats compared to the control group. The increase of collagen fibers in the stroma was accompanied by invasion of macrophages, neuronal degeneration and the presence of mast cells. The increase of macrophages and mast cells are suggestive of inflammatory situation prevailing in the bladder connective stroma of diabetic animals with six and twenty weeks (Figure 7).

Although the thickness of the epithelium may reflect bladder distention in relation to detrusor muscle hypofunction due to autonomic neuronal degeneration observed in diabetic animals, widespread mitochondrial alterations observed are suggestive of metabolic disorders such as accumulation of free radicals. The ultrastructural alterations observed in muscle fibers (myocytes) could suggest a metabolic alteration and consequent dysfunction of the detrusor muscle ${ }^{18}$.

There was an increase in the thickness of the lamina propria of the bladder in diabetic rats. This finding may be related to collagen deposition that occurs at different rates in diabetic animals and in healthy animals. There is evidence in the literature that there is an increase in the proportion of type III collagen in diabetic animals than in healthy animals, young or old.

There was an increase in thickness of bladder detrusor smooth muscle in diabetic rats. This increase was more pronounced with time twenty weeks duration of diabetes that occurs demonstrating a significant hypertrophy of the detrusor muscle. Pitre et al. ${ }^{11}$ reported an increase in total mass of the bladder and the lumen cross-sectional area according to time in diabetic rats from five weeks demonstrating induction of hypertrophy and dilatation of the detrusor correlated with the duration of diabetes.

Regarding the urethra there was a decrease in the thickness of the urothelium diabetic rats after six and twenty weeks of induction of experimental diabetes. However, the total thickness of the urethra increased considerably, which may be due to hypertrophy of the urethral muscle layer.

Gasbarro et al. ${ }^{20}$ carried out histological analysis of the urethra in rats after six weeks of induction of diabetes and found that the external sphincter showed edema and fibrosis periurethral muscle layer. This infiltration of collagen leads to a sharp discontinuity of the urethral striated muscle structure compared with the control group. The bladder wall of diabetic rats showed edema and vasculopathy. These associated with dilated vascular channels changes may contribute to noticeable changes in the microenvironment of the smooth muscle and connective tissue structure bladder.

Studies evaluating the morphology of the lower urinary tract in diabetic rats reveal neuropathological findings as vacuolization of ganglion cells of the bladder wall, the presence of sympathetic neurons giants hipocromaticas ganglion cells, demyelination and axonal changes ${ }^{21-22}$.

In the present study we observed increased presence of macrophages and mast cells in the perivascular area, mainly in the urinary bladder of diabetic animals with longer induction, sometimes accompanied by the presence of vacuoles in some myelinated nerve fibers present in the lamina propria. These findings are suggestive of an inflammatory process in the stroma of the bladder in the diabetic state may be related to oxidative stress associated with characteristics complications of diabetes 
mellitus. The severity of bladder injuries is also correlated to the duration of diabetes as observed in other studies ${ }^{12,20}$. Most studies, however, have not correlated morphological bladder lesions with the duration of diabetes mellitus and urodynamic evaluation.

\section{Conclusions}

Diabetes induces urethropathy is characterized by increased peak urethral pressure during voiding associated with increased total thickness of layer of urethral rats. Changes in the urethra function and morphological components should be included as a possible impairment factor in studies evaluating lower urinary tract dysfunction induced by diabetes.

\section{References}

1. Liu G, Daneshgari F. Alterations in neurogenically mediated contractile responses of urinary bladder in rats with diabetes. Am J Physiol Renal Physiol. 2005 Jun;288(6):1220-26. doi: 10.1152/ ajprenal.00449.2004.

2. Kaplan, SA, Te AE, Blaivas JG. Urodynamic findings in patients with diabetic cystopathy. J Urol. 1995 Feb;153(2):342-4. PMID: 7815578.

3. Yoshimura N, Chancellor MB, Andersson KE, Christ GJ. Recent advances in understanding the biology of diabetes-associated bladder complications and novel therapy. BJU Int. 2005 Apr;95(6):733-8. doi: 10.1111/j.1464-410X.2005.05392.x.

4. Yenilmez A, Ozçifçi M, Aydin Y, Turgut M, Uzuner K, Erkul A. Effect of high dose thiamine on detrusor contractily. Acta Diabetol. 2006 Dec;43:(4):103-8. doi: 10.1007/s00592-006-0223-5.

5. Rodrigues AA Jr, Suaid HJ, Tucci S Jr, Fazan VPS, Foss MC, Cologna AJ, Martins ACP. Long term evalution of functional and morphological bladder alteration on alloxan induced diabetes and aging. Experimental study in rats. Acta Cir Bras. 2008;23(1):53-8. doi: 10.1590/s0102-865020080000700010.

6. Poladia DP, Bauer JA. Functional, structural, and neuronal alterations in urinary bladder during diabetes: investigations of a mouse model. Pharmacology. 2005 May;74(2):84-94. doi:10.1159/000083962.

7. Uvelius B. Detrusor smooth muscle in rats with alloxan-induced diabetes. J Urol. 1986 Oct;136(4):949-52. PMID: 3761467.

8. Andersson PO, Malmgren A, Uvelius B. Cystometrical and in vitro evaluation of urinary bladder function in rats with streptozotocininduced diabetes. J Urol. 1988 Jun;139(6):1359-62. PMID: 3373615.

9. Malmgren A, Andersson PO, Uvelius B. Bladder function in rats with short- and long-term diabetes; effects of age and muscarinic blockade. J Urol. 1989 Dec;142(6):1608-14. PMID: 2585645

10. Lincon J, Haven A, Sawyer M, Burnstock G. The smooth muscle of rat bladder in the early stages of streptozotocin-induced diabetes. $\mathrm{Br}$ J Urol. 1984 Feb;56(1):24-30. PMID: 6697101.

11. Pitre DA, Wallace LJ, Bauer JA. Time-dependent urinary bladder remodeling in the streptozotocin-induced diabetic rat model. Acta Diabetol. 2002 Apr; 39(1):23-7. PMID: 12043935.

12. Rizk DE, Padmanabhan RK, Tariq S, Shafiullah M, Ahmed I. Ultrastructural morphological abnormalities of the urinary bladder in streptozotocin-induced diabetic female rats. Int Urogynecol J Pelvic Floor Dysfunct. 2006 Feb;17(2):143-54. PMID: 16021327.

13. Daneshgari F, Liu G, Imrey PB. Time dependent changes in diabetic cystopathy in rats include compensaded and decompensated bladder function. J Urol. 2006 Jul;176(1):380-6. doi: 10.1016/s00225347(06)00582-9.

14. Liu G, Lin, YH, Yamada Y, Daneshgari F. External urethral sphincter activity in diabetic rats. Neurourol Urodyn. 2008;27(5):429-34. doi: 10.1002/nau.20543.

15. Torimoto K, Fraser MO, Hirao Y, de Groat WC, Chancellor MB and Yoshimura N. Urethral dysfunction in diabetic rats. J Urol. 2004 May;171(5):1959-64. doi: 10.1097/01.ju.0000121283.92963.05.

16. Torimoto K, Hirao Y, Matsuyoshi H, de Groat WC, Chancellor MB, Yoshimura N. Alpha -1-adrenergic mechanism in diabetic urethral dysfunction in rats. J Urol. 2005 Mar;173(3):1027-32. doi: 10.1097/01.ju.0000146268.45662.36.

17. Yang Z, Dolber PC, Fraser MO. Diabetic urethopathy compounds the effect of diabetic cystopathy. J Urol. 2007 Nov;178(5):2213-9. doi: 10.1016/juro.2007.06.042.

18. Rodrigues AA Jr, Suaid HJ, Tucci S Jr, Fazan VP, Foss MC, Cologna AJ, Martins AC. Long term evaluation of functional and morphological bladder alterations on alloxan-induced diabetes and aging: experimental study in rats. Acta Cir Bras. 2008;23(1):53-8. doi: 10.1590/s0102-86502008000700010.

19. Eika B, Levin RM, Longhurst PA. Comparison of urinary bladder function in rats with hereditary diabetes insipidus, streptozotocininduced diabetes mellitus, and nondiabetic osmotic diuresis. J Urol. 1994 Feb;151(2):496-502. PMID: 8283565

20. Gasbarro G, Lin DL, Vurbic D, Quisno A, Kinley B, Daneshgari F, Damaser MS. Voiding function in obese and type 2 diabetic female rats. Am J Physiol Renal Physiol. 2010 Jan;298(1):72-7. doi: 10.1152/ajprenal.00309.2009.

21. Elbadawi A, Diokono AC, Millard RJ. The aging bladder: morphology and urodynamics. World J Urol. 1998;16(1):10-34. PMID: 9775413.

22. Kolta MG, Wallace LJ, Gerald MC. Streptozocin-induced diabetes affects rat urinary bladder response to autonomic agents. Diabetes. 1985 Sep;34(9):917-21. PMID: 4029511.

\section{Correspondence:}

Ana Beatriz Gomes de Souza Pegorare

Faculdade de Medicina de Ribeirão Preto

Divisão de Urologia

Departamento de Cirurgia e Anatomia

Avenida Bandeirantes, 3900

14048-900 Ribeirão Preto - SP Brasil

Tel./Fax: (55 16)3602-2453

beatrizgomes@usp.br

Received: Feb 20, 2014

Review: April 22, 2014

Accepted: May 19, 2014

Conflict of interest: none

Financial source: none

${ }^{1}$ Research performed at Laboratory of Surgical Technique and Experimental Surgery, Department of Surgery and Anatomy, Faculty of Medicine of Ribeirao Preto, University of Sao Paulo (FMRP-USP), Ribeirao Preto-SP, Brazil. Part of PhD degree thesis, Postgraduate Program in Surgery, Department of Surgery and Anatomy, FMRP-USP. Tutor: Haylton Jorge Suaid. 